\title{
Clinical features of diabetic patients referred by general physicians due to less ophthalmic examinations
}

This article was published in the following Dove Press journal:

Clinical Ophthalmology

16 July 2014

Number of times this article has been viewed

\section{Yayoi Otsu \\ Masato Matsuoka \\ Kayako Matsuyama \\ Tetsuya Nishimura}

Department of Ophthalmology, Kansai Medical University, Takii

Hospital, Osaka, Japan
Correspondence: Masato Matsuoka Department of Ophthalmology, Kansai Medical University Takii Hospital, 10-15 Fumizono-cho Moriguchi, Osaka 570-8507, Japan

$\mathrm{Tel}+81669921001$ extension 3322

Fax +81669928263

Email matsuokm@takii.kmu.ac.jp
Background: The purpose of this study was to determine the clinical features of patients with type 2 diabetes, and less ophthalmic examinations, referred by general physicians to ophthalmologists.

Methods: The medical charts of 327 patients with type 2 diabetes referred to our department from general physicians were reviewed. A detailed medical history was taken and a complete ophthalmic examination was performed for all patients. The patients were divided into two groups, ie, those with a history of missing ophthalmic examinations for more than a year (noncompliant group) and those with no previous ophthalmic examinations (never-examined group). Serum levels of glycosylated hemoglobin and creatinine, estimated glomerular filtration rate, and urine albumin/creatinine ratio were obtained from medical records.

Results: Of the 327 patients, 102 had diabetic retinopathy (31.2\%), with a mean best-corrected visual acuity of $0.037 \pm 0.36 \log$ MAR (logarithm of the minimum angle of resolution) units. Of the 327 patients, 203 were in the never-examined group and 124 were in the noncompliant group. The incidence of diabetic retinopathy in the noncompliant group was significantly higher than that in the never-examined group $(P<0.001)$. Best-corrected visual acuity in the noncompliant group was significantly worse than in the never-examined group $(P=0.004)$. Glycosylated hemoglobin levels and estimated glomerular filtration rate in the noncompliant group were significantly lower than in the never-examined group $(P<0.001$ and $P<0.003$, respectively); serum creatinine levels and urine albumin/creatinine ratio were significantly higher $(P=0.020$ and $P=0.001$, respectively). The severity of the diabetic retinopathy was significantly correlated with compliance in terms of ophthalmic examinations and with urine albumin/creatinine ratio (multiple regression analysis, $P=0.047$ and $P<0.001$, respectively).

Conclusion: Our results show that diabetic patients referred from general physicians due to less ophthalmic examinations generally have good visual acuity, but one third of them have diabetic retinopathy. A history of missing ophthalmic examinations and albuminuria are risk factors for diabetic retinopathy.

Keywords: diabetic retinopathy, general physician, ophthalmologists, albuminuria

\section{Introduction}

Diabetic retinopathy (DR) is a serious microvascular complication of diabetes mellitus and a major cause of decreased vision in working-aged adults. ${ }^{1}$ The risk of a severe decrease in vision can be reduced by effective control of serum glucose and by regular ophthalmic examinations for early detection and appropriate treatment, such as laser photocoagulation. ${ }^{2-6}$ Guidelines for frequency of ophthalmic examinations for early detection and timely treatment have been published. ${ }^{7}$ However, several studies have 
reported that more than one third of diabetic patients did not follow the guidelines, ${ }^{8}$ and low compliance with ophthalmic examinations was the cause of decreased vision even if vitreous surgery had been performed. ${ }^{9}$ Therefore, better communication with general physicians is necessary to encourage better compliance with regular ophthalmic follow-up examinations. This has been shown to be true in many patients who have less regular ophthalmic examinations even when they have regular general examinations for diabetes. ${ }^{10}$

Several large cohort studies have reported three major risk factors for DR, ie, duration of diabetes, ${ }^{11-13}$ circulating glycosylated hemoglobin $\left(\mathrm{HbA}_{1 \mathrm{c}}\right)$ levels, ${ }^{11-15}$ and blood pressure. ${ }^{16-20}$ However, to the best of our knowledge, little has been reported on the clinical features of diabetic patients referred from general physicians due to less ophthalmic examinations. Thus, the purpose of this study was to determine the features of diabetic patients referred from general physicians to the Kansai Medical University Takii Hospital for evaluation of the eyes.

\section{Patients and methods}

\section{Patients}

The procedures used in this study conformed to the tenets of the Declaration of Helsinki. A total of 327 consecutive patients with type 2 diabetes referred from general physicians to the Department of Ophthalmology, Kansai Medical University Takii Hospital (Osaka, Japan) between April 2009 and March 2012 were studied. Patients who were referred from ophthalmologists or continued to have regular ophthalmic examinations at least once a year were excluded.

At the initial examination, all patients were interviewed regarding their medical history, including any ocular diseases. All underwent a standard ophthalmic examination, including best-corrected visual acuity (BCVA), refraction, slit-lamp examination, and fundus examination with dilated pupils. BCVA was determined as the decimal visual acuity and then converted to logMAR (logarithm of the minimum angle of resolution) units for statistical analysis. In cases where the eyes had different BCVA values, the eye with the lower BCVA was used.

The patients were divided into two groups, ${ }^{9}$ ie, those with a history of missing ophthalmic examinations at our hospital or other ophthalmic clinics for more than a year (noncompliant group) and those without any previous ophthalmic examination since the onset of diabetes mellitus (never-examined group).

\section{Classification of DR and diabetic status}

The stage of DR was determined by ophthalmoscopy and fluorescein angiography. The patients were classified according to severity of DR, ie, no apparent DR, mild to moderate nonproliferative DR, severe nonproliferative DR, and proliferative DR. ${ }^{21}$ In cases where both eyes had different stages of DR, the eye with the more severe stage was selected for statistical analysis.

Serum $\mathrm{HbA}_{1 \mathrm{c}}$ levels (defined by the Japan Diabetes Society: JDS), serum creatinine, estimated glomerular filtration rate (eGFR), urinary levels of creatinine ( $\mathrm{uCre}$ ) and albumin (uAlb), and urine albumin/creatinine ratio (uACR) were obtained from the medical records and taken on the day closest to the first visit to our clinic.

\section{Statistical analysis}

The results are expressed as the mean \pm standard deviation. Mann-Whitney $U$-tests were used to determine whether differences in the different sets of values were significant. A comparison of the incidence of DR between the noncompliant group and never-examined group was made by computing odds ratios and 95\% confidence intervals (CIs). Multiple linear regression analysis was used to analyze the association between severity of DR and clinical parameters. Differences were considered to be statistically significant when the $P$-value was $<0.05$. Statistical analysis of the data was performed using IBM ${ }^{\circledR}$ SPSS $^{\circledR}$ Statistics for Windows version 22 (IBM Corporation, Armonk, NY, USA).

\section{Results}

\section{Patient characteristics}

Of the 327 patients, 124 (38\%) belonged to the noncompliant group and 203 (62\%) to the never-examined group (Table 1). The mean overall patient age was $60.0 \pm 14.0$ years, that of the noncompliant group was $63.0 \pm 14.0$ years, and that of the never-examined group was $58.1 \pm 13.8$ years. The noncompliant group was significantly older than the never-examined group $(P=0.001)$.

The mean BCVA for all patients was $0.037 \pm 0.36$ $\log$ MAR units, that of the noncompliant group was $0.15 \pm 0.53$ $\log$ MAR units, and that of the never-examined group was $-0.03 \pm 0.18 \log$ MAR units. The BCVA in the neverexamined group was significantly better than that in the noncompliant group $(P=0.004)$.

\section{Incidence of DR}

Of the 327 patients, 102 were diagnosed with DR $(31.2 \%$, 95\% CI 0.26-0.36; Table 2), 81 with mild to moderate nonproliferative DR $(24.8 \%, 95 \%$ CI $0.20-0.30)$, eleven with severe nonproliferative DR $(3.4 \%, 95 \%$ CI $0.02-0.06)$, and ten with proliferative DR $(3.1 \%, 95 \%$ CI $0.01-0.06)$. The incidences of DR, mild to moderate nonproliferative 
Table I Clinical characteristics

\begin{tabular}{lllll}
\hline Category & Total & Noncompliant & Never-examined & P-value* \\
\hline Patients $(\mathrm{n})$ & 327 & 124 & 203 & $\mathrm{~N} / \mathrm{A}$ \\
Age (years) & $60.0 \pm \mid 4.0$ & $63.0 \pm \mid 4.0$ & $58.1 \pm \mid 3.8$ & $0.00 \mathrm{I}$ \\
Sex (male/female) & $229 / 98$ & $86 / 38$ & $143 / 60$ & $\mathrm{~N} / \mathrm{A}$ \\
BCVA (logMAR) & $0.037 \pm 0.36$ & $0.15 \pm 0.53$ & $-0.03 \pm 0.18$ & 0.004 \\
\hline
\end{tabular}

Notes: Values are shown as the mean \pm standard deviation. $* P$-value between noncompliant group and never-examined group (Mann-Whitney $U$-test). Abbreviations: BCVA, best-corrected visual acuity; logMAR, logarithm of the minimum angle of resolution; N/A, not applicable.

$\mathrm{DR}$, and proliferative DR in the noncompliant group were significantly higher than the corresponding values in the never-examined group (odds ratios 3.4, 3.3, and 15.8, respectively; $P<0.001, P<0.001$, and $P<0.002$; Table 2). However, the incidence of severe nonproliferative DR was not significantly different between the groups.

\section{Blood chemistry}

$\mathrm{HbA}_{1 \mathrm{c}}$ levels and eGFR in the noncompliant group were significantly lower than the corresponding values in the never-examined group $\left(\mathrm{HbA}_{1 \mathrm{c}} 8.1 \% \pm 2.0 \%\right.$ versus $9.1 \% \pm 2.4 \%, P<0.001$; eGFR $72.3 \pm 25.1 \mathrm{~mL} / \mathrm{min}$ versus $84.2 \pm 29.6 \mathrm{~mL} / \mathrm{min}, P=0.003$; Table 3 ). In contrast, levels of serum creatinine, uAlb, and uACR in the noncompliant group were significantly higher (serum creatinine $1.0 \pm 0.8 \mathrm{mg} / \mathrm{dL}$ versus $0.9 \pm 1.2 \mathrm{mg} / \mathrm{dL}, P=0.020 ; \mathrm{uAlb}$ $365 \pm 1,140 \mathrm{mg} / \mathrm{L}$ versus $188 \pm 989 \mathrm{mg} / \mathrm{L}, P=0.002$; and uACR, $428 \pm 1,291 \mathrm{mg} / \mathrm{g} \cdot$ Cre versus $176 \pm 638 \mathrm{mg} / \mathrm{g} \cdot \mathrm{Cre}$, $P=0.001$ ). The level of uCre was not significantly different between the two groups.

\section{Multiple regression analysis}

Multiple regression analysis showed that the severity of DR was significantly correlated with compliance with ophthalmic examination and uACR (adjusted $R^{2}=0.11 ; P=0.047$ and $P<0.001$, respectively; Table 4), but not with age, BCVA, $\mathrm{HbA}_{1 \mathrm{c}}$, or eGFR.

\section{Discussion}

Our results show that diabetic patients referred by general practitioners due to less ophthalmic examinations had four major characteristics. First, the patients had reasonably good visual acuity (Table 1). Patients generally visited an ophthalmologist without being referred by their general physician if they had some degree of eye symptoms, such as loss of visual acuity or floaters.

Second, the incidence of DR was $31.2 \%$ in our study (Table 2), which is higher than in large two populationbased studies in Japan (the Hisayama and Funagata studies), that reported incidences of $16.9 \%$, and $23.0 \%$, respectively, in diabetic patients. ${ }^{12,22}$ In the USA, the National Health and Nutrition Examination Survey between 2005 and 2008 found that $28.5 \%$ of adults with diabetes had DR. ${ }^{23}$ A recent meta-analysis showed the global prevalence of DR to be $35 \%$ in diabetic individuals. ${ }^{24}$ The incidence of DR in our study is similar to the global average, but higher than those in the large studies in Japan and the USA. The reason for this may be that the patients attending our hospital have more severe diabetes than those seen at local clinics.

Third, a habit of missing ophthalmic examinations was a risk factor for DR. The incidence of DR in the noncompliant group was higher than that in the never-examined group (Table 2), and the severity of DR was significantly correlated with noncompliance (Table 4). A number of studies have already reported that missing ophthalmic examinations leads to progression of DR and low vision. ${ }^{8-10}$ The reason for this may be that noncompliant patients have had diabetes for longer than patients without previous ophthalmic examinations. However, it is generally difficult to determine the exact duration of type 2 diabetes in patients with the disease. This is also a reason for the poorer values for indices of renal

Table 2 Prevalence of diabetic retinopathy

\begin{tabular}{lllll}
\hline Category & Total (\%) & Noncompliant (\%) & Never-examined (\%) & P-value* (OR) \\
\hline Total $(n)$ & 327 & 124 & 203 & N/A \\
DR $(n)$ & $102(3 I .2)$ & $59(47.6)$ & $43(2 I .2)$ & $<0.00 I(3.4)$ \\
M-NPDR & $8 I(24.8)$ & $48(38.7)$ & $33(16.3)$ & $<0.00 I(3.3)$ \\
S-NPDR & $11(3.4)$ & $2(1.6)$ & $9(4.4)$ & $0.29(0.4)$ \\
PDR & $10(3.1)$ & $9(13.8)$ & $1(0.6)$ & $0.002(I 5.8)$ \\
\hline
\end{tabular}

Note: *P-value from the comparison of prevalence of DR between never-examined group and noncompliant group.

Abbreviations: DR, diabetic retinopathy; M-NPDR, mild to moderate nonproliferative diabetic retinopathy; OR, odds ratio; S-NPDR, severe nonproliferative diabetic retinopathy; PDR, proliferative diabetic retinopathy; N/A, not applicable. 
Table 3 Biochemistry results

\begin{tabular}{|c|c|c|c|c|}
\hline Category & Total & Noncompliant & Never-examined & $P$-value* \\
\hline $\mathrm{HbA}_{\mathrm{Ic}}(\%)$ & $8.8 \pm 2.3$ & $8.1 \pm 2.0$ & $9.1 \pm 2.4$ & $<0.001$ \\
\hline sCre (mg/dL) & $0.9 \pm I . I$ & $1.0 \pm 0.8$ & $0.9 \pm 1.2$ & 0.020 \\
\hline eGFR (mL/min) & $79.8 \pm 28.6$ & $72.3 \pm 25.1$ & $84.2 \pm 29.6$ & 0.003 \\
\hline uCre (mg/dL) & $106 \pm 78.5$ & $99.8 \pm 71.2$ & $110 \pm 82.3$ & 0.428 \\
\hline uAlb (mg/L) & $251 \pm 1,046$ & $365 \pm 1,140$ & $188 \pm 989$ & 0.002 \\
\hline uACR (mg/g.Cre) & $266 \pm 931$ & $428 \pm 1,291$ & $176 \pm 638$ & 0.001 \\
\hline
\end{tabular}

Notes: Mean \pm standard deviation. $* P$-value between noncompliant group and never-examined group (Mann-Whitney $U$-test).

Abbreviations: $\mathrm{HbA}_{\mathrm{lc}}$, glycosylated hemoglobin; sCre, serum creatinine; eGFR, estimated glomerular filtration rate; uCre, urinary creatinine; uAlb, urinary albumin; uACR, urine albumin/creatinine ratio.

function (eGFR, uAlb, and uACR) in the noncompliant group than in the never-examined group (Table 3 ).

Fourth, we found that albuminuria was also a risk factor for DR in our patients. The severity of DR was significantly correlated with $\mathrm{UACR}$, but not with eGFR or HbA $\mathrm{Hc}_{\mathrm{c}}$ (Table 4). uACR is commonly used as an index of albuminuria. ${ }^{25} \mathrm{Chen}$ et al and Moriya et al reported that microalbuminuria was a more useful biomarker than moderately decreased eGFR in predicting the severity of DR in patients with type 2 diabetes. ${ }^{26,27} \mathrm{We}$ found that $\mathrm{HbA}_{1 \mathrm{c}}$ in the never-examined group was higher than that in the noncompliant group (Table 3), although severity of DR was not correlated with $\mathrm{HbA}_{1 \mathrm{c}}$ (Table 4). The reason for this may be that the patients in the never-examined group had not had a general examination.

This study has some limitations. First, we did not examine blood pressure, which is a major risk factor for DR. However, blood pressure may not be an appropriate parameter for assessment of DR, because it can be easily altered by hypotensive drugs. Second, this was a retrospective study at a university hospital, so further studies are needed with larger and more diverse samples.

In summary, our results show that diabetic patients referred from general physicians due to less ophthalmic examinations have good visual acuity but one third have DR.

Table 4 Multiple regression analysis of severity of diabetic retinopathy

\begin{tabular}{lll}
\hline $\begin{array}{l}\text { Independent } \\
\text { variable }\end{array}$ & $\boldsymbol{\beta}^{*}$ & $\boldsymbol{P}$-value \\
\hline Group & \\
Age & -0.14 & 0.047 \\
BCVA & 0.03 & 0.683 \\
$\mathrm{HbA}$ & & 0.466 \\
eGFR & 0.05 & 0.053 \\
uACR & 0.13 & 0.954 \\
\hline
\end{tabular}

Notes: Adjusted $R^{2}=0.1$ I for final BCVA in multiple regression $(P<0.00 \mathrm{I})$; *standard partial regression coefficient; " noncompliant group or never-examined group. Abbreviations: $\mathrm{BCVA}$, best-corrected visual acuity; $\mathrm{HbA}_{\mathrm{lc}}$, glycosylated hemoglobin; eGFR, estimated glomerular filtration rate; $\mathrm{UACR}$, urine albumin/creatinine ratio.
In addition, a history of missing ophthalmic examinations and albuminuria are risk factors for DR in these patients. Our results suggest that ophthalmologists need to inform not only their diabetic patients but also general physicians about the need for regular follow-up ophthalmic examinations.

\section{Disclosure}

The authors report no conflicts of interest in this work.

\section{References}

1. Mohamed Q, Gillies MC, Wong TY. Management of diabetic retinopathy: a systematic review. JAMA. 2007;298:902-916.

2. [No authors listed]. Photocoagulation treatment of proliferative diabetic retinopathy. Clinical application of Diabetic Retinopathy Study (DRS) findings, DRS Report Number 8. The Diabetic Retinopathy Study Research Group. Ophthalmology. 1981;88:583-600.

3. [No authors listed]. Photocoagulation for diabetic macular edema. Early Treatment Diabetic Retinopathy Study report number 1. Early Treatment Diabetic Retinopathy Study research Group. Arch Ophthalmol. 1985;103:1796-1806.

4. Klein R, Klein BE, Moss SE, Davis MD, DeMets DL. The Wisconsin Epidemiologic Study of Diabetic Retinopathy. IX. Four-year incidence and progression of diabetic retinopathy when age at diagnosis is less than 30 years. Arch Ophthalmol. 1989;107:237-243.

5. Klein R, Klein BE, Moss SE, Davis MD, DeMets DL. The Wisconsin Epidemiologic Study of Diabetic Retinopathy. X. Four-year incidence and progression of diabetic retinopathy when age at diagnosis is 30 years or more. Arch Ophthalmol. 1989;107:244-249.

6. Wong TY, Mwamburi M, Klein R, et al. Rates of progression in diabetic retinopathy during different time periods: a systematic review and meta-analysis. Diabetes Care. 2009;32:2307-2313.

7. American Diabetes Association. Standards of medical care in diabetes 2012. Diabetes Care. 2012;35 Suppl 1:S11-S63.

8. Schoenfeld ER, Greene JM, Wu SY, Leske MC. Patterns of adherence to diabetes vision care guidelines: baseline findings from the Diabetic Retinopathy Awareness Program. Ophthalmology. 2001;108:563-571.

9. Itoh-Tanimura M, Hirakata A, Itoh Y, et al. Relationship between compliance with ophthalmic examinations preoperatively and visual outcome after vitrectomy for proliferative diabetic retinopathy. Jpn J Ophthalmol. 2012;56:481-487.

10. Newcomb PA, Klein R, Massoth KM. Education to increase ophthalmologic care in older onset diabetes patients: indications from the Wisconsin Epidemiologic Study of Diabetic Retinopathy. J Diabetes Complications. 1992;6:211-217.

11. [No authors listed]. Intensive blood-glucose control with sulphonylureas or insulin compared with conventional treatment and risk of complications in patients with type 2 diabetes (UKPDS 33). UK Prospective Diabetes Study (UKPDS) Group. Lancet. 1998;352:837-853. 
12. Miyazaki M, Kubo M, Kiyohara Y, et al. Comparison of diagnostic methods for diabetes mellitus based on prevalence of retinopathy in a Japanese population: the Hisayama Study. Diabetologia. 2004;47:1411-1415.

13. Kawasaki R, Tanaka S, Tanaka S, et al. Incidence and progression of diabetic retinopathy in Japanese adults with type 2 diabetes: 8 year follow-up study of the Japan Diabetes Complications Study (JDCS). Diabetologia. 2011;54:2288-2294.

14. [No authors listed]. The effect of intensive treatment of diabetes on the development and progression of long-term complications in insulindependent diabetes mellitus. The Diabetes Control and Complications Trial Research Group. N Engl J Med. 1993;329:977-986.

15. Ohkubo Y, Kishikawa H, Araki E, et al. Intensive insulin therapy prevents the progression of diabetic microvascular complications in Japanese patients with non-insulin-dependent diabetes mellitus: a randomized prospective 6-year study. Diabetes Res Clin Pract. 1995;28:103-117.

16. [No authors listed]. Tight blood pressure control and risk of macrovascular and microvascular complications in type 2 diabetes: UKPDS 38. UK Prospective Diabetes Study Group. BMJ. 1998;317:703-713.

17. Chaturvedi N, Sjolie AK, Stephenson JM, et al. Effect of lisinopril on progression of retinopathy in normotensive people with type 1 diabetes. The EUCLID Study Group. EURODIAB controlled trial of lisinopril in insulin-dependent diabetes mellitus. Lancet. 1998;351:28-31.

18. Chaturvedi N, Porta M, Klein R, et al. Effect of candesartan on prevention (DIRECT-Prevent 1) and progression (DIRECT-Protect 1) of retinopathy in type 1 diabetes: randomised, placebo-controlled trials. Lancet. 2008;372:1394-1402.
19. Sjolie AK, Klein R, Porta M, et al. Effect of candesartan on progression and regression of retinopathy in type 2 diabetes (DIRECT-Protect 2): a randomised placebo-controlled trial. Lancet. 2008;372:1385-1393.

20. Chew EY, Ambrosius WT, Davis MD, et al. Effects of medical therapies on retinopathy progression in type 2 diabetes. $N$ Engl J Med. 2010;363:233-244.

21. Wilkinson CP, Ferris FL 3rd, Klein RE, et al. Proposed international clinical diabetic retinopathy and diabetic macular edema disease severity scales. Ophthalmology. 2003;110:1677-1682.

22. Kawasaki R, Wang JJ, Wong TY, Kayama T, Yamashita H. Impaired glucose tolerance, but not impaired fasting glucose, is associated with retinopathy in Japanese population: the Funagata study. Diabetes Obes Metab. 2008;10:514-515.

23. Zhang X, Saaddine JB, Chou CF, et al. Prevalence of diabetic retinopathy in the United States, 2005-2008. JAMA. 2010;304:649-656.

24. Yau JW, Rogers SL, Kawasaki R, et al. Global prevalence and major risk factors of diabetic retinopathy. Diabetes Care. 2012;35:556-564.

25. National Kidney Foundation. K/DOQI clinical practice guidelines for chronic kidney disease: evaluation, classification, and stratification. $\mathrm{Am}$ J Kidney Dis. 2002;39:S1-S266.

26. Chen YH, Chen HS, Tarng DC. More impact of microalbuminuria on retinopathy than moderately reduced GFR among type 2 diabetic patients. Diabetes Care. 2012;35:803-808.

27. Moriya T, Tanaka S, Kawasaki R, et al. Diabetic retinopathy and microalbuminuria can predict macroalbuminuria and renal function decline in Japanese type 2 diabetic patients: Japan diabetes complications study. Diabetes Care. 2013;36:2803-2809.
Clinical Ophthalmology

\section{Publish your work in this journal}

Clinical Ophthalmology is an international, peer-reviewed journa covering all subspecialties within ophthalmology. Key topics include: Optometry; Visual science; Pharmacology and drug therapy in eye diseases; Basic Sciences; Primary and Secondary eye care; Patient Safety and Quality of Care Improvements. This journal is indexed on

Submit your manuscript here: http://www.dovepress.com/clinical-ophthalmology-journal

\section{Dovepress}

PubMed Central and CAS, and is the official journal of The Society of Clinical Ophthalmology (SCO). The manuscript management system is completely online and includes a very quick and fair peer-review system, which is all easy to use. Visit http://www.dovepress.com/ testimonials.php to read real quotes from published authors. 